$4.3 \%, 2.2 \%, 2.2 \%$, and $0.7 \%$ respectively. These findings resulted in the change from curative to palliative aims in $5.0 \%$ and increasing in adjuvant therapy up to $24.5 \%$. stage III, primary tumor size, and the presence of pelvic and/or paraaortic lymphadenopathy shown by whole abdomen CT were all determined to be significantly prognostic in the recurrence rate. Conclusion: This study implied the potential benefits of CT scans as important for proper staging of primary diagnosis, and determination of disease extent with proper treatment planning to reach the aim of improving outcome.

Poster (C22)

Cervical Cancer

https://doi.org/10.3802/jgo.2021.32.S1.C22

\section{Radical hysterectomy in cervical cancer during pregnancy: a case report}

\section{Hiro Hidaya Danial Nasution, ${ }^{1, *}$ Kartiwa Hadi Nuryanto ${ }^{2}$}

'Faculty of Medicine, Obstetric \& Gynecology Department, University of Indonesia, Dr. Cipto Mangunkusumo Hospital, Jakarta, Indonesia (hiro_nasution@yahoo.com)

${ }^{2}$ Faculty of Medicine, University of Indonesia, Dr. Cipto Mangunkusumo Hospital, Gynecologic Oncology Division, Jakarta, Indonesia

Objective: The management of cervical cancer during pregnancy represents a challenge for the physician, and it is influenced mostly by gestational age at diagnosis, stage of disease, and patient's desire of maintaining the pregnancy. Our current case was a pregnant woman with stage IB2 cervical cancer who underwent radical hysterectomy and pelvic lymphadenectomy during the cesarean section.

Methods: A 42-year-old woman, G5P3A1, at 32 weeks of gestation came to our hospital complaining vaginal bleeding for about 3 months. Gynecologic examination revealed a 2 $\mathrm{cm}$ exophytic cervical lesion without vaginal and parametrial involvement. A cervical biopsy was performed that revealed an invasive, poorly differentiated, squamous cell carcinoma of the cervix. An ultrasonographic examination confirmed the presence of a cervical lesion with the size of $3 \times 2 \times 2 \mathrm{~cm}$ with no parametrial involvement. The cesarean section was performed at 35 weeks of gestation with a live baby girl, 2,530 g, AS 9/10. The procedure was followed by radical hysterectomy, left salpingo-oophorectomy, right salpingectomy, right ovarian transposition and pelvic lymphadenectomy.

Results: The final histopathology result revealed an invasive, moderately differentiated, squamous cell carcinoma of the cervix with no metastasis to the parametrial and lymph node, and also free margin to the vaginal border. We did not give any adjuvant treatment based on our analysis to the histopathology result. After 7 months of observation, no sign of recurrence was found.

Conclusion: Management of cervical cancer during pregnancy is influenced by gestational age, stage of disease, patient's desire to maintain her pregnancy with careful evaluation and counselling of the patient. We report a case of 42-year-old pregnant woman with cervical cancer stage IB2 with successfully treated by radical hysterectomy and pelvic lymphadenectomy during cesarean section.

Poster (C23)

Cervical Cancer

https://doi.org/10.3802/jgo.2021.32.S1.C23

\section{Recurrent cervical carcinoma presenting as periumbilical abdominal wall mass treated with wide excision followed by reconstruction using surgical mesh: a rare case report}

Moh Nailul Fahmi, ${ }^{1,{ }^{*}}$ Andi Darma Putra, ${ }^{1}$ Trifonia Pingkan Siregar ${ }^{2}$

'Gynecology Oncology Division, Department of Obstetrics and Gynecolgy, Faculty of Medicine, Dr. Cipto Mangunkusumo, Universitas Indonesia, Jakarta, Indonesia (fahmi0995g@gmail.com)

${ }^{2}$ Department of Radiology, Faculty of Medicine, Dr. Cipto Mangunkusumo Hospital, Universitas Indonesia, Jakarta, Indonesia

Objective: The recurrence of cervical cancer most commonly presents as nodal diseases in pelvic or paraaortic lymph nodes. The prevalence of recurrence in the periumbilical abdominal wall is low. The management depends on patient's condition. We herein report a rare case of the abdominal wall recurrence with diagnosis and management.

Methods: A case report of diagnosis and management. Results: A 56-year-old woman with history of radical hysterectomy followed by external beam radiotherapy and brachytherapy for stage IIA of poorly differentiated adenocarcinoma of the cervix one year ago. She complained about a mass in her abdomen. Physical examination revealed a $3 \times 3 \mathrm{~cm}$ solid mass in the umbilical region. The MRI result showed a $3 \times 2.3 \times 1.9 \mathrm{~cm}$ intramuscular solid mass with necrotic component in the right abdominal rectus, $4.1 \mathrm{~cm}$ apart from the cutis, and was suspected as metastasis. We performed a laparotomy adhesiolysis, extirpation of solid tumor mass in periumbilical, and then reconstruction by prosthesis (mesh) insertion. The histopathology result corresponded with metastatic carcinoma from the cervix. The patient's condition was good after surgery.

Conclusion: Periumbilical abdominal wall recurrence of cervical carcinoma is unusual and can be treated with wide excision followed by reconstruction using surgical mesh. 